\title{
A Rare Case of Keratoacanthoma of the Palm: Special Considerations when Treating Keratoacanthoma of the Hand
}

\author{
Ofelia Leroux ${ }^{*}$, Y-Vu Robert Van ${ }^{2}$ and Ryan Engdahl ${ }^{1}$ \\ ${ }^{l}$ Department of Surgery, Division of Plastic and Reconstructive Surgery, NYC Health + Hospitals Harlem Hospital, Columbia University, New York, New \\ York, USA \\ ${ }^{2}$ Department of Surgery, Division of Plastic and Reconstructive Surgery, New York Presbyterian Hospital, Columbia University, Weill Cornell University, \\ New York, New York, USA
}

\begin{tabular}{l} 
A R T I C L E I N F O \\
\hline Article history: \\
Received: 2 August, 2021 \\
Accepted: 16 August, 2021 \\
Published: 2 September, 2021 \\
\hline Keywords: \\
Keratoacanthoma \\
palm \\
hand \\
non-follicular
\end{tabular}

\begin{abstract}
A B S T R A C T
Keratoacanthoma (KA) is a low-grade, rapidly growing skin tumor which is thought to originate from the pilosebaceous unit from hyperkeratosis of the infundibulum and are often thought to originate on hairbearing skin or sun-exposed surfaces. There are very scarce reports demonstrating they may occur in other areas such as mucous membranes or soles of the feet. We present a rare case of palmar KA in a 65-year-old female with no known antecedent history. Surgical excision was performed with complete removal of the tumor. Following surgical excision, the pathology of the irregularly elevated $0.7 \times 0.2 \mathrm{~cm}$ lesion revealed a keratoacanthoma. The patient remained without evidence of recurrence at one year follow-up. We believe there is only one other report of isolated palmar KA to date in the literature. We review relevant literature on hand KA.
\end{abstract}

Background

Keratoacanthoma (KA) is a low-grade, rapidly growing skin tumor which is thought to originate from the pilosebaceous unit from hyperkeratosis of the infundibulum. KA commonly occurs in sunexposed areas in middle-aged adults, with peak incidence being 50-69 years of age and a higher incidence in males. Multiple risk factors such as exposure to UV radiation, chemical carcinogens, immunosuppression, genetic predispositions (i.e., p53 mutations), human papillomavirus, recent trauma or surgery have been linked to developing KA [1]. An important caveat of KA is that it is considered by some to be a highly differentiated form of squamous cell carcinoma (SCC) of the skin, requiring distinct treatment options for patients [1]. Although Keratoacanthomas are often thought to originate on hair-bearing skin or sun-exposed surfaces, there are very scarce reports demonstrating they can occur such as mucous membranes or soles of the feet [2]. We present a rare case of a palmar lesion and review literature on special considerations when treating KA of the hand.

\section{Case Presentation}

A 65-year-old right hand dominant female with past medical history of diabetes mellitus, hypertension, hyperlipidemia, asthma, presented with a $0.5 \times 0.5 \mathrm{~cm}$, pedunculated, pruritic, intermittently hemorrhagic mass on the palmar aspect of the right hand in the thenar eminence as can be seen in (Figure 1A). The patient reported that the mass appeared two months prior to presentation with rapid growth and denied any history of trauma or surgery to the area. There were no additional masses or regional lymphadenopathy evident on physical exam. Surgical excision was performed with complete removal of the lesion. Pathology results demonstrated an irregularly elevated lesion and revealed a keratoacanthoma. The incision was able to be closed primarily and healed well with no post-operative complications. The patient remained without evidence of recurrence at one year follow-up as can be seen in (Figure 1B).

${ }^{*}$ Correspondence to: Ofelia Leroux, M.D., Department of Surgery, Division of Plastic and Reconstructive Surgery, NYC Health + Hospitals Harlem Hospital, Columbia University, 506 Lenox Avenue, 10037, New York, New York, USA; Tel: 2129393529; E-mail: lerouxo@nychhc.org, ofelialeroux@gmail.com 


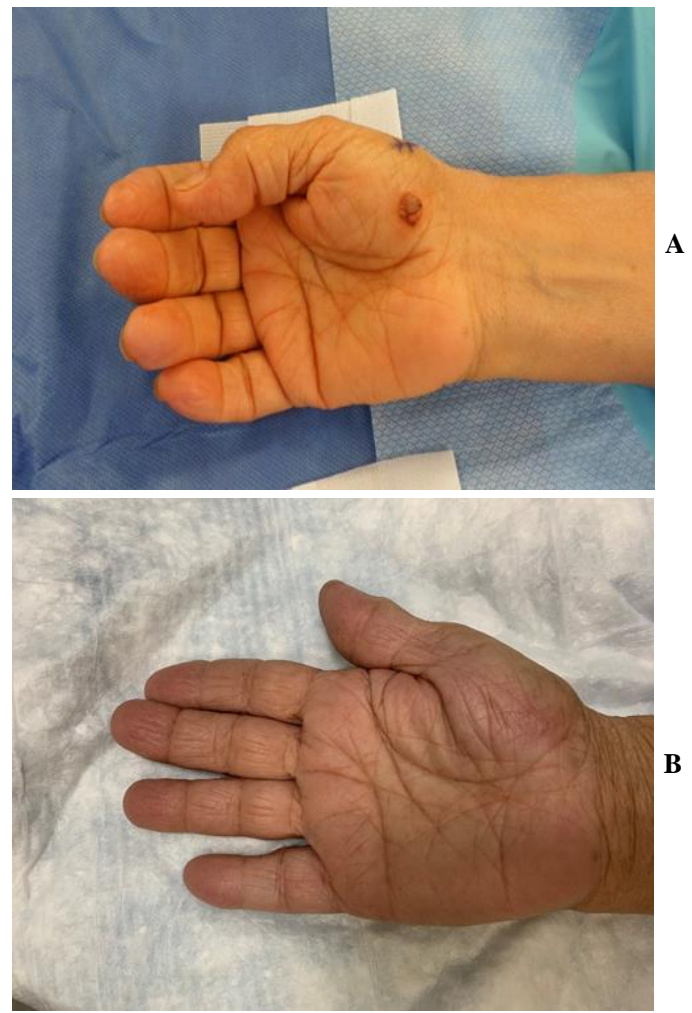

Figure 1: A) Right palm lesion on thenar eminence. B) Post-operative site healed after surgical excision.

\section{Discussion}

Keratoacanthoma is a benign, rapidly growing dome-shaped skin tumor with a central keratinous plug (umbilication). Keratoacanthoma is a prototype of pseudo-malignant skin tumors and usually forms within a four-to-six-week period [1,3]. Histologically, the mass has an acute angle with a central crater with overlying epithelium and then typically normal appearing superficial epithelium on the lateral aspect of the tumor. The central crater is filled with proliferating keratin and epithelial cells, which results in a chronic inflammatory response [4]. An important aspect of this tumor is that it can also be considered an abnormal variant SCC of the skin. As such, it is paramount to distinguish both pathologies. For example, both lesions can present as a firm red nodule with potential invasion and keratin scale. Some unique clinical features of KA are that lesions can spontaneously regress and heal. Conversely, KA lesions can also be extremely aggressive, undergo rapid growth and cause local tissue destruction and metastasis. Recommended treatment for a solitary KA is surgical resection with $4 \mathrm{~mm}$ margins. However, often KA may grow unpredictably and pose challenges for adequate surgical resection and may lead to significant disfiguring lesions. Other treatment options then include methotrexate, intralesional 5-FU and Acitretin [5]. Overall, KA has an excellent prognosis following complete removal with surgical excision.

Although it is known that true keratoacanthomas regress and may be treated with observation alone, it is not as commonly done. The minority of keratoacanthomas are treated this way; the majority are instead treated with surgical excision, intra-lesion therapeutics, topical or systemic therapies. This is due to the uncertainty of adequate diagnosis of the KA as compared to SCC and the possibility of malignant transformation into
SCC. Even pathological analysis may be inconclusive and thus the clinical exam and history are the most pertinent for diagnosis. The history of growth plateau or regression are the most important for this distinction. Keratoacanthoma of the hand presents a unique problem. Depending on the location of the KA, the resultant scarring from either surgical, medical or conservative management may have long term consequences in regard to cosmesis and function, with scarring, joint contracture, and bony destruction being a major concern. In a case series from 2003, a single surgeon reported on his treatment of 19 KA's of which 5 were on the hand. In his experience, he observed all 5 hand KA's with complete resolution and satisfactory cosmesis of all of them [6]. In a literature review of subungual keratoacanthomas, observation may be detrimental to patients as the extrinsic pressure of the growth is known to cause destruction of the distal phalanx and thus observational treatment is not recommended. In these cases, excision or curettage is the treatment of choice with the possibility of a distal amputation in cases of recurrence $[7,8]$.

Keratoacanthoma of the hand must be evaluated carefully. If a sound diagnosis is made, then treatment modalities should be chosen based on the location of it to optimize functional preservation. In locations of the hand that may predispose to bony destruction, prompt treatment should be undertaken in order to prevent bony loss. In locations along joint lines, a clinical decision must be made as to the proper treatment. Observation and eventual resolution are said to have the best results in regard to scarring and less predisposed to contracture though there have been no blinded studies to compare the scarring to either treatment modality. In locations that are not predisposed to bony or contracture issues, any of the aforementioned treatments may be used pending the clinician's judgement. Keratoacanthoma of the hand, though not a distinct pathological entity from elsewhere on the body, does present a different set of potential functional problems and thus treatment should be tailored to optimize cosmetic, bony, and functional outcomes.

\section{Author Contributions}

OL is the first author and wrote the initial draft of the manuscript and subsequent edits. All other coauthors were involved in the writing, revisions and refining of the manuscript. All the authors read and approved the final manuscript for submission.

\section{Funding}

None.

\section{Availability of Data and Materials}

All datasets on which the conclusions of the manuscript rely are presented in the main paper.

\section{Competing Interests}

\section{None.}




\section{REFERENCES}

1. Zito PM, Scharf R (2021) Keratoacanthoma. In: StatPearls [Internet]. Treasure Island (FL): StatPearls Publishing. [Crossref]

2. Cohn I, Kanat IO (1985) Keratoacanthoma of the foot. Case presentation and review of the literature. J Am Podiatr Med Assoc 75: 653-655. [Crossref]

3. Cohen MM (1957) Keratoacanthoma. CA Cancer J Clin 7: 206. [Crossref]
4. Zargaran M, Baghaei F (2014) A clinical, histopathological and immunohistochemical approach to the bewildering diagnosis of keratoacanthoma. J Dent (Shiraz) 15: 91-97. [Crossref]

5. Girijala RL, Kwak Y, Wright D, Goldberg LH (2018) Solitary Palmar Keratoacanthoma: Case Report. Cureus 10: e2331. [Crossref]

6. Griffiths RW (2004) Keratoacanthoma observed. Br J Plast Surg 57: 485-501. [Crossref]

7. Patel MR, Desai S (1989) Subungual keratoacanthoma in the hand. $J$ Hand Surg Am 14: 139-142. [Crossref]

8. Pellegrini Jr VD, Tompkins A (1986) Management of subungual keratoacanthoma. J Hand Surg Am 11: 718-724. [Crossref] 